\title{
Tertulias Pedagógicas Dialógicas: dando voz al aprendizaje de los estudiantes en la educación superior
}

Pedagogical Dialogic Gatherings: giving a voice to the learning process of higher education students

Recibido: 19 de marzo 2021, evaluado: 27 de marzo 2021, aceptado: 15 de abril 2021

Cristina Laorden-Gutiérrez

http://orcid.org/0000-0001-7770-5234

cristina.laorden@ cardenalcisneros.es

Universidad de Alcalá, Alcalá de Henares, España

Mercedes Foncillas-Beamonte

http://orcid.org/0000-0002-4052-1607

mercedes.foncillas@ cardenalcisneros.es

Universidad de Alcalá, Alcalá de Henares, España 


\section{Resumen}

El presente artículo analiza el impacto de las Tertulias Pedagógicas Dialógicas (TPD) como práctica educativa de éxito en un contexto universitario, favoreciendo así otras formas de aprender alejadas de las clases magistrales. Durante cuatro cursos académicos-se ha analizado el desarrollo de las intervenciones de 87 estudiantes de tercer curso del grado de Educación Social. Por un lado, se diseñó un cuestionario, cuyos datos se analizaron de forma cuantitativa y, por otro lado, se llevó a cabo un análisis cualitativo de los relatos de los estudiantes en las sesiones de TPD y de entrevistas en profundidad, estableciéndose cuatro categorías de análisis. Los resultados muestran la contribución de esta práctica a la futura actividad profesional del alumnado, dotándole de instrumentos para implementarla en diferentes contextos sociales. También muestran su contribución en el fomento de su capacidad crítica y reflexiva, en la relación con el entorno, en el fomento de modelos basados en el aprendizaje entre iguales y en las reflexiones que se realizan en torno a la lectura. Este artículo pretende aportar algunas evidencias de la experiencia de TPD en la educación superior contribuyendo así a aumentar datos científicos que avalen esta actuación de éxito en este nivel educativo.

Palabras clave: tertulia Pedagógica Dialógica, escucha activa, reflexión, aprendizaje dialógico, Educación Social

\section{Summary}

This article analyses the impact of Pedagogical Dialogic Gatherings (PDG) as a successful educational approach in a university context, thus favouring other learning methods that move away from traditional lectures. During four academic years, the interventions of 87 third year Social Education students have been analysed. On the one hand, a survey whose data were analysed quantitatively was created; on the other hand, the students' accounts in the PDG sessions and in-depth interviews were qualitatively analysed and four categories of analysis were established. The results show how this method can contribute to the future professional activity of the students, providing them with the necessary tools to implement it in different social contexts. The results also show its contribution to the development of their critical and reflective capacity, to the relationship with the environment, to the promotion of models based 
on peer learning and to the reflections on reading. This article aims to provide some evidence of the PDG experience in higher education, therefore contributing to increase scientific data that support this successful method at this level of education.

Keywords: pedagogical Dialogic Gathering, active listening, reflection, dialogic learning, Social Education

\section{Introducción}

Durante ocho cursos hemos implementado experiencias de aprendizaje de diálogo igualitario en el grado de educación social, a través de Tertulias Pedagógicas Dialógicas (TPD), desde la necesidad de cambiar la enseñanza universitaria y coincidiendo con Rekalde, Alonso, Arandia, Martínez y Zarandona (2014) cuando señalan que la educación superior debe ir más allá del conocimiento de las distintas disciplinas y que estas se deben acercar al entorno en el que se encuentra el alumnado. Freire (1997) señalaba que la dialogicidad es una exigencia de la propia naturaleza humana y el educador democrático debe hacer uso de ella.

En este sentido, Bruner (1997) establece que la educación no está aislada del entorno, siempre tiene consecuencias sociales y económicas, que se deben entender como facilitadoras de cambio.

Cuando hablamos de metodologías que ayudan a avanzar a los estudiantes universitarios en su aprendizaje debemos pensar en dos tipos de actuación diferente, como señala Flecha (2015), las prácticas o acciones que obtienen excelentes resultados y las acciones que obtienen excelentes resultados en muy diversos contextos, a los que llama acciones de éxito. Diferentes estudios de la comunidad científica internacional han demostrado que las tertulias dialógicas ayudan a que el estudiante aprenda mejor y que mejora la convivencia en el centro educativo (García-Carrión, López de Aguileta, Padrós y Ramis-Salas, 2020; Grañeras, Díaz Caneja y Gil, 2011; Malagón Moreno y González López, 2018). En educación, los estudios científicos nos hacen críticos y si nos alejamos de la ciencia y nos basamos en intuiciones o experiencias personales también nos alejamos del espíritu crítico, indispensable en toda transformación social, como señala Roca (2016).

El aprendizaje dialógico considera que aprendemos a partir de la interacción comunitaria con otros generando acuerdos intersubjetivos entre las personas participantes 
(Aubert, Flecha, García, Flecha y Racionero, 2008; García-Carrión et al., 2020; Habermas, 1992). Lo fundamental en su definición es la importancia de las interacciones diversas a partir de la igualdad y el diálogo que necesitamos las personas para aprender. Todos contribuimos al conocimiento de los demás. Esta interacción está por encima de la lectura o la escucha (Álvarez, González y Larrinaga, 2012). En este sentido, Bruner (1997), establece que cuando se aprende a interpretar, a dar sentido a lo que otras personas sienten y lo que otras personas expresan, se consolida el aprendizaje y su proceso, y si estas interacciones, además, se producen entre iguales, este aprendizaje será más sólido.

Al utilizar el diálogo en la lectura, como señala Aguilar Ródenas (2017), se favorece un aprendizaje reflexivo desde un posicionamiento crítico de la realidad. Cada participante en las TPD comparte el libro estableciendo inferencias con el entorno en el que vive y entre todos van construyendo su aprendizaje. Además, el significado de lo que cada uno va leyendo y compartiendo lo define el propio lector a través de su experiencia, sus objetivos y sus conocimientos previos (Bruner, 1997: Del Pino-Sepúlveda, Del Pino-Sepúlveda y PincheiraFuentealba, 2016). El diálogo supone un instrumento para fomentar la reflexión, la comunicación, la transformación tanto del entorno, como de la manera de entender la realidad (García-Carrión et al., 2020). Además, Freire (1970) nos recuerda que sin el diálogo no hay comunicación y sin esta no hay verdadera educación.

En general, las experiencias llevadas a cabo, como la que presentan Gabaldón-Estevan (2016) en la universidad de Valencia, nos muestran una alta satisfacción del alumnado en la educación superior con esta práctica. Por su parte, Rekalde et al., (2014) llevaron a cabo la experiencia de tertulias dialógicas en un grupo de primer curso de Educación Social en una universidad del País Vasco, destacando en sus conclusiones la positividad en sus intenciones formativas de unir el mundo académico y el mundo de la vida.

Bonell, Martínez-González y Rodríguez-Fernández (2019) nos muestran su experiencia en grupos de educación social y trabajo social. Los estudiantes deben vivir lo que están aprendiendo y con la implementación de las tertulias dialógicas se consigue siempre que se sigan fielmente los principios del aprendizaje dialógico.

En el terreno de la formación inicial de los educadores sociales, consideramos que debe contribuir a la inclusión social y al éxito en el desarrollo personal y académico de los estudiantes o beneficiarios de diferentes programas sociales relacionados con educación. Roca (2019) señala que esta formación tiene un gran impacto en los resultados de los alumnos y es imprescindible cuando se unen calidad científica y humana. Además, al igual que Chocarro de 
Luis (2013), defiende la formación dialógica, ya que mejora la calidad del aprendizaje y del profesorado en la docencia. En este mismo sentido Flecha, Racionero, Tintoré y Arbós, (2014) señalan, que el alumnado universitario, en su formación inicial, cuando se implementa alguna actuación de éxito, aprende de forma más profunda y reflexiva.

A pesar de todo lo expuesto anteriormente, seguimos necesitando evidencias en la educación superior. Palomares-Montero y Gabaldón-Estevan (2016) han analizado las distintas contribuciones de los docentes universitarios al proceso de transformación de la educación superior con sus innovaciones metodológicas. Se centran en dos de ellas, tertulias dialógicas y grupos interactivos, que son propuestas que ponen al estudiante en el centro del proceso de enseñanza-aprendizaje y estimulan las relaciones de diálogo y colaboración. Señalan que las referencias en la universidad todavía son escasas y sin investigaciones que demuestren su generalización en esta etapa.

Objetivos de esta investigación:

1. Analizar la percepción que el alumnado tiene sobre la influencia de las TPD en su aprendizaje.

2. Conocer el número de intervenciones que el alumnado realiza durante las sesiones de las TPD.

3. Analizar el discurso del alumnado en las sesiones de tertulias realizadas.

\section{Método}

\section{Sujetos participantes}

La muestra de estudio fueron 87 estudiantes de tercer curso del Grado de Educación Social. Esta muestra está formada 75 alumnas y 12 alumnos. Con la participación de tres alumnas del programa Erasmus.

\section{Procedimiento}

Dos han sido las asignaturas implicadas: Medio Escolar y Educación de Personas Adultas, pertenecientes al primer cuatrimestre de tercer curso del grado de Educación Social.

La recogida de datos se ha llevado a cabo a lo largo de cuatro cursos académicos, desde 2015-16 hasta 2018-19. En este periodo se organizaron, cada curso, dos grupos de alumnos (A y B) que se reunían cada quince días para llevar a cabo la tertulia dialógica, cada uno con sus 
aportaciones sobre la lectura convenida entre todos y ejerciendo de moderar uno de los estudiantes en cada ocasión. La asignación del alumnado en estos grupos siempre ha sido aleatoria, con la excepción, siempre que fuera posible, que en los dos debía haber representación de ambos sexos y de alumnado procedente de programas de intercambio internacional. En las sesiones participaban las dos profesoras, y los libros elegidos fueron: $L a$ pedagogía del oprimido (Freire 1970) y A la sombra de este árbol (Freire, 1997). Todas las sesiones (42) se grabaron con el permiso del alumnado para su análisis.

\section{Instrumentos de evaluación}

Para la recogida de datos se utilizaron tres instrumentos:

- Cuestionario sobre percepción de los estudiantes tras su experiencia de esta buena práctica, elaborado por las investigadoras. Al tratarse de una encuesta sobre percepciones, que no ha sido diseñada para medir constructos psicológicos, no existen datos psicométricos previos. Para validar su fiabilidad, presentamos el resultado del coeficiente Alfa de Cronbach, $\alpha=.91$.

El cuestionario está estructurado en tres bloques. En el primero, encontramos variables referidas a cómo se han sentido los estudiantes y la valoración de elementos importantes en las sesiones como puntualidad, respeto mutuo, escucha, expresión verbal, lectura, así como compartir la lectura con otros, expresión en libertad; el segundo bloque se destina al aprendizaje y vida profesional y el tercero a las competencias generales y específicas conseguidas. Las preguntas del cuestionario, en los distintos bloques, son estructuradas y sus respuestas se basan, bien en una escala de tipo Likert de 1 a 5, o bien en respuesta múltiple, con cuatro opciones desde nada valorado a lo más valorado.

- Registro de datos de participación e intervención en las sesiones. Todas las sesiones de tertulias dialógicas se grabaron en vídeo para su posterior análisis. Posteriormente se analizó el discurso de los alumnos y su percepción en función de las categorías que se describen posteriormente.

- Entrevista en profundidad. Se llevó a cabo con una submuestra de 12 estudiantes, con los que se realizaron entrevistas semiestructuradas para profundizar en los datos obtenidos a través del cuestionario descrito anteriormente.

Para estructurar el análisis de la información obtenida en las entrevistas en profundidad y en las sesiones grabadas, se definieron una serie de categorías. Así, se fue clasificando el 
contenido de los relatos de los estudiantes para, posteriormente, poder ver el porcentaje de las referencias realizadas en cada categoría de análisis.

\section{Las categorías:}

- Actividad profesional, referida a habilidades y estrategias para el desempeño profesional.

- Capacidad crítica y reflexión, para contrastar opiniones y tener un pensamiento propio, valorando y escuchando los planteamientos del resto.

- Aprendizaje, referido a ideas, teorías, competencias, actitudes, que partiendo de la lectura del libro se fomenta entre todos. Se resuelven dudas entre iguales y se establecen interacciones con otras materias, reforzando así los conocimientos ya adquiridos.

- Relación con el entorno. El aprendizaje no solo se produce en el aula, sino también en contextos familiares y profesionales y las lecturas y reflexiones que tienen lugar en el aula, se comparten con amigos, familia, conocidos.

\section{Análisis de datos y resultados}

Se realizaron análisis cualitativos y cuantitativos.

Para el tratamiento de datos cualitativos, tanto de las sesiones realizadas como de las entrevistas, se han seguido las pautas de la metodología comunicativa crítica (Gómez, Latorre, Sánchez y Flecha, 2006). Esta metodología facilita el análisis de las contribuciones de todas las personas participantes, teniendo en cuenta todas las opiniones, imprescindible en el contexto dialógico en el que estamos.

Los datos obtenidos, en el cuestionario se analizaron de forma cuantitativa, realizando análisis descriptivos de frecuencias y porcentajes de los ítems de cada bloque.

\section{Datos de análisis del cuestionario}

En cuanto a la percepción de la experiencia, un 92\% del alumnado manifiesta haberse sentido bien o muy bien, durante las sesiones de tertulias.

Entre los motivos para este sentimiento, destaca como lo más valorado el considerar que toda opinión sea válida (91.9\%) y tan solo un 3.4\% ha elegido la opción: haberse sentido incómodo por no gustarle hablar en público. El resto de los motivos presentan, también, valores altos. 
En cuanto a la valoración de los elementos que se indican en la Tabla 1, señalar que todos ellos han sido valorados con porcentajes altos, destacando el respeto mutuo entre todos los miembros del grupo, un 95.4\%, y la capacidad de escucha durante las sesiones, un $90.8 \%$.

\section{Tabla 1}

Elementos organizativos valorados

\section{ELEMENTOS VALORADOS}

\begin{tabular}{ll}
\hline Puntualidad & $73.5 \%$ \\
Respeto entre los miembros & $95.4 \%$ \\
Capacidad de escucha & $90.8 \%$ \\
Orden en las intervenciones & $73.5 \%$ \\
Expresión verbal & $64.4 \%$ \\
Lectura realizada & $75.8 \%$
\end{tabular}

El alumnado percibe que el mayor aprendizaje logrado durante el desarrollo de las TPD ha sido la libertad de expresión (93.1\%) seguido del diálogo igualitario (89.6\%) como se observa en la tabla 2.

\section{Tabla 2}

Porcentajes de los ítems relacionados con el aprendizaje durante la experiencia

APRENDIZAJE DURANTE LA EXPERIENCIA

\begin{tabular}{lc}
\hline Libertad de expresión & $93.1 \%$ \\
Diálogo igualitario & $89.6 \%$ \\
Aprendizaje entre todos & $87.4 \%$ \\
Reflexión & $79.3 \%$ \\
Conocer más a los compañeros & $58.6 \%$ \\
Capacidad de escucha & $82.8 \%$
\end{tabular}

En cuanto a cómo perciben el aprendizaje para implementarlo en su futura vida profesional (Tabla 3), para un $95.4 \%$ el respeto y el diálogo igualitario son las mejores aportaciones, seguido de no imponer tu opinión, un $93.1 \%$. 
Tabla 3

Aportaciones de las tertulias al aprendizaje para la vida profesional

APORTACIONES A TU APRENDIZAJE PARA TU VIDA PROFESIONAL

\begin{tabular}{lc}
\hline Aprender a escuchar & $89.6 \%$ \\
No imponer tu opinión & $\mathbf{9 3 . 1 \%}$ \\
Respeto y diálogo igualitario & $\mathbf{9 5 . 4 \%}$ \\
Fomentar la transformación a través de la reflexión & $85.0 \%$ \\
Participar y superar retos & $67.8 \%$ \\
Interesarme por la lectura de autores clásicos de la Educación & $390 \%$ \\
\hline
\end{tabular}

En la valoración que el alumnado realiza sobre su participación en las TPD, lo más puntuado ha sido la asistencia $(83.9 \%)$ y la escucha activa (70.1\%). Por otro lado, lo menos puntuado ha sido la profundidad de la reflexión, (22.9\%) seguido de la relación de lo leído con experiencias personales (36.8\%). Consideramos que los estudiantes se muestran críticos con su participación en las tertulias.

El 96.6\% del alumnado considera que las TPD se basan en una metodología que debe conocer como educadores/as sociales, y argumentan motivos como la mejora de la práctica profesional y la reflexión personal, un 43,7\%.

\section{Resultados sobre el número de intervenciones en las sesiones de tertulias dialógicas}

En las sesiones de tertulias, grabadas, se han contabilizado un total de 1.172 intervenciones (Tabla 7). Destaca la reflexión y la capacidad crítica con un $63.5 \%$ del total de las intervenciones, seguido de la categoría relacionada con el entorno del alumnado fuera del aula, tanto familiar como de iguales, con un $18 \%$. En cuanto a sus intervenciones directas, basadas en el aprendizaje en el aula, resolución de dudas, relación con otras asignaturas, suponen un $8.44 \%$. Siendo las intervenciones menos presentes aquellas relativas al ejercicio de su futuro profesional $(6.3 \%)$. 


\section{Tabla 7}

Intervenciones del alumnado y porcentajes según cursos, libro de lectura y categorías

\begin{tabular}{|c|c|c|c|c|c|c|}
\hline $\begin{array}{l}\text { Libro } \\
\text { de lectura }\end{array}$ & Curso & Profesión & Reflexión & Entorno & $\begin{array}{l}\text { Aprendizaj } \\
\mathrm{e}\end{array}$ & Total \\
\hline Pedagogía & 2015-16 & $14(7.5 \%$ & $121(65.1 \%)$ & $22(11.8 \%)$ & $29(15.59$ & 186 \\
\hline del & (grupos A y & ) & & & $\%)$ & \\
\hline \multirow[t]{3}{*}{ Oprimido } & B) & & & & & \\
\hline & $2017-18$ & $35(9.61$ & $245(67.3 \%)$ & $32(8.79 \%)$ & $52(14.28$ & 364 \\
\hline & $\begin{array}{l}\text { (grupos A y } \\
\text { B) }\end{array}$ & $\%)$ & & & $\%)$ & \\
\hline A la Sombra & 2016-17 & $9(3.91 \%$ & $159(69.13)$ & $47(20.43 \%)$ & $15(6.52 \%$ & 230 \\
\hline de este & (grupos A y & ) & & & ) & \\
\hline \multirow[t]{4}{*}{ Árbol } & B) & & & & $\rightarrow$ & \\
\hline & 2018-19 & $16(4.08$ & $219(55.86$ & $110(28.06$ & $47(11.98$ & 392 \\
\hline & (grupos A y & $\%)$ & $\%)$ & $\%)$ & $\%)$ & \\
\hline & B) & & & & $\Rightarrow$ & \\
\hline TOTAL & 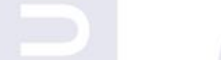 & $74(6.3 \%$ & $744(63.5 \%)$ & $211(18 \%)$ & $99(8.44 \%$ & 1172 \\
\hline & & & & & ) & \\
\hline
\end{tabular}

\section{Resultados sobre la observación de las sesiones y las entrevistas}

A continuación, se muestra el análisis cualitativo realizado a partir de la transcripción de las sesiones y de las entrevistas semiestructuradas según las categorías de análisis anteriormente descritas.

\section{Actividad profesional}

En las intervenciones desarrolladas durante las sesiones de las tertulias, un $6.31 \%$ hace referencia directa a la actividad profesional. El alumnado argumenta aspectos como:

"Veo valores muy importantes, que yo relacionaba mucho con nuestro trabajo, la perseverancia, seguir con la persona. No sé, lo relacionaba mucho con la educación social". "La relación entre educador / educando debe ser recíproca... Hay muchas cosas que el educando puede aportar al educador". 
El futuro contexto profesional está presente en los estudiantes. La igualdad y la escucha activa enlazan con la propuesta de educación problematizadora de Freire (1970) y cala en el alumnado, así como la humildad, la igualdad, argumentando que estas han de ser las líneas que guiarán su profesión: "Yo te educo a ti, (...) soy humilde, soy tolerante y tú eres igual como al final nos retroalimentamos".

$\mathrm{Al}$ analizar las entrevistas, se observa que todo el alumnado entrevistado coincide en señalar a las TPD como una buena herramienta para implementar en su vida profesional, argumentando esta afirmación con aspectos como, por ejemplo:

“(...) no solo hay que trabajarlo con estudiantes. Se puede trabajar con muchísimos colectivos, con niños, con personas mayores, con personas con discapacidad”.

Otra alumna señala: "En mi práctica profesional es muy importante saber escuchar a los demás, saber debatir, decir algo asertivamente”. En los diversos colectivos con los que van a intervenir precisarán, a lo largo de la implementación de diferentes programas educativos, la escucha activa. Así, señalan que implementarían esta metodología en su vida profesional, justificando su respuesta con los siguientes argumentos:

"Si tú sientes que te escuchan y tienes libertad de expresión, al final como que te sientes mejor contigo mismo, y eso es como la clave para el cambio y la transformación de las personas con las que trabajamos", "La conciencia crítica que se genera”. Otros argumentos: "Creo que se puede generar un clima muy bueno entre las personas que están ahí si se hace bien, conseguir lo mismo que he conseguido yo: respetar el turno de palabra, escuchar diferentes puntos de vista, abrir un poco tu mente, fomentar la lectura de una manera más creativa".

El alumnado valora las TPD para el desarrollo de su profesión y relacionan esta práctica con el ejercicio de su futura actividad profesional desde el diálogo igualitario, en el que todos aprenden de todos.

\section{Capacidad crítica y reflexión}

Del total de las intervenciones del alumnado durante las sesiones, un $63.5 \%$ de estas se realizaban basándose en la reflexión que les producía la lectura del libro. Algunas de estas reflexiones son:

Tras la lectura de un capítulo de Pedagogía del oprimido, una alumna señala: “(...) Es una constante lucha, no se puede tirar la toalla. Pienso que es difícil, hay personas que 
creen que están en una zona de confort, pero si quieren cambiar... Quiero salir de ahí y tengo que seguir luchado".

Otra alumna reflexiona sobre la lectura realizada señalando: “Estos libros, en realidad lo que nos han hecho, es un poco despertarnos de nuestro letargo, de decir, llevamos años y años con lo mismo y a lo mejor yo no era consciente... y es que en vosotros puede estar el cambio".

En la argumentación que da, además de su profunda reflexión, la transformación que la lectura a través de las TPD le ha producido. Por otro lado, Habermas (1992) señala que la acción comunicativa, que se desarrolla en diferentes contextos, la argumentación, el diálogo, la reflexión, fomenta la madurez.

Otra estudiante reflexiona sobre la educación y señala:

“(... si no fomentamos que los niños piensen por sí mismos, que puedan decidir en un aspecto u otro... nos quieren inculcar que las cosas vayan por un camino, no se plantean otras alternativas".

En las entrevistas realizadas, las respuestas de las estudiantes hacen referencia a su opinión crítica sobre las TPD, aspectos positivos y negativos de las mismas, a la reflexión sobre el impacto en sus vidas y a la transformación experimentada. Hay que señalar, que todas coinciden en la importancia de compartir la lectura, que toda opinión es válida, que su conocimiento se amplía con el de las otras personas, la igualdad entre todas las personas del grupo. Algunas de las argumentaciones, las más comunes a todas, que dan en sus respuestas son:

"Para mí ha sido un descubrimiento, antes leía los libros y no los compartía con nadie, ahora los comparto con mis compañeros, incluso con mi familia y amistades", "Algunos testimonios personales le dan más vida al libro y eso también te hace compartirlo y crearte una visión más amplia de lo leido". "Porque tu profesora está a tu nivel, puedes debatir con ella también".

En cuanto a los aspectos positivos y negativos, también hay una coincidencia en la mayoría de las entrevistadas, señalando como aspectos positivos: 
"Poder validar todo tipo de opiniones, que nadie sienta que su opinión no es igual de válida que la de otra persona”, "Fomentar la escucha activa y el respeto", "interacciones entre las personas".

Como aspectos negativos, la mayoría expresó no ocurrírsele ninguno, pero algunas señalaron dos:

“Adaptación al tiempo, a veces me gustaría hacer más”.

En cuanto al impacto en sus vidas y su transformación, si bien es cierto que en todos ha dejado una huella, no hay unanimidad en sus argumentaciones. Recogemos algunas de estas:

“Lo he extrapolado mucho, mucho a mi vida personal y sí que ha habido momentos de estar leyendo y pensar en mí (...), no puedo seguir leyendo porque me estoy dando cuenta de la realidad que estoy viviendo”, "Te sientes más igual, estas entre iguales”.

La transformación que estos estudiantes han experimentado con las TPD, podemos observarlo en las siguientes respuestas:

“A veces sales de la tertulia pensando tanto en lo que has hablado, sales como reflexionando", "Yo creo que me ha mejorado como persona (...). Yo no soy la misma que en $1^{\circ}$, y creo que esto tiene mucho que ver, del efecto que me ha hecho respetar, tener que escuchar, todo es válido, lo tuyo, lo mío y lo de la otra persona, yo antes ni escuchaba. Creo que he mejorado como persona".

\section{Aprendizaje}

Del total de las intervenciones del alumnado durante las sesiones, un $18.0 \%$ de estas se realizaban basándose en el aprendizaje que les producía la lectura del libro con sus compañeras y compañeros. Estas intervenciones bien se dirigían a relacionar lo tratado en la TPD con otras asignaturas o bien se centraban en resolver dudas que otros compañeros y compañeras tenían, siempre sin imponer opiniones, partían de la consideración que toda opinión es válida.

Algunas de estas argumentaciones son:

"Recordar lo visto en Educación de personas adultas" 
En esta asignatura se hacía referencia a diferentes aspectos planteados en los libros leídos de Freire.

Teniendo como referencia el diálogo igualitario y la horizontalidad, presente en Freire, argumenta:

"El educador se convierte en educando, porque también está aprendiendo de las experiencias de los demás". "Se necesita ser libre, no partir de la base de que somos ignorantes y siempre hay alguien que sepa más, sino que cada uno sabe cosas en ciertos ámbitos y se pueden enriquecer mutuamente las personas y no tener miedo a decir nuestra verdad".

Otra estudiante relaciona lo leído en La sombra de este árbol con la vista a una residencia de personas mayores con la profesora de otra asignatura, recordando el comentario de un usuario de la residencia: "Es que a mi siempre me hubiera gustado estudiar historia. Yo le dije: nunca es tarde, pero él me contestó: no hija ya no sirvo para eso". Las erróneas teorías del deterioro cognitivo todavía se encuentran arraigadas en sectores de la población, produciéndose una discriminación basada en la edad.

En las entrevistas se trataba de conocer si las TPD fomentaban el aprendizaje, tanto en las dos asignaturas implicadas, como en el resto de las asignaturas. En este apartado, nuevamente todas las alumnas entrevistadas coincidieron en que a través de las tertulias se fomenta el aprendizaje, siendo más reflexivo y duradero. Estas son algunas de sus argumentaciones:

"De esta forma nos enriquecemos todos. Incluso vosotros también como profesores y además estamos todos al mismo nivel", "Yo ahora me tengo que enfrentar a leerme un libro, expresar una opinión, saber extraer lo que estoy pensando, plasmarlo (...) Tampoco nos educan en reflexionar críticamente”, "Yo creo que las competencias que desarrollas en las tertulias, no las dejas en las tertulias, sino que sales con ellas. Al final esto te va a servir tanto para otra asignatura, como para tu vida".

Preguntadas sobre su aprendizaje en la última sesión, algunas de las respuestas fueron: 
"Me quedaron como dos ideas claras, la primera y principal fue que el ser humano ya está determinado. Que la educación no tiene que basarse en un aprendizaje memorístico, sino que tiene que ser a través de preguntas", "La situación de opresor / oprimido en la sociedad, en general, es la misma situación que en la escuela, en la educación de profesor / educando", "Concebir al educando como una vasija, (...) veo que con muchos profesores he sido esa vasija que me llenaban”.

Una alumna, después de haber leído A la sombra de este árbol (Freire 1997) señala: “QQué entendemos por contenidos indispensables? Porque cuando estaba leyendo el libro, pensaba es indispensable la convivencia, es indispensable que no aislemos a alguien en clase, es indispensable que asumamos que hay diferencias. A mí no me lo han transmitido nunca. Yo lo he aprendido por la educación que he recibido en casa, pero en el Instituto, al menos en el mío, no ha habido nadie que me diga, si tienes un problema con un compañero puedes venir al equipo de orientación (...). Tenemos unas prioridades que no son tan prioridades y a lo mejor lo esencial han transmitido nunca”.

Valora la importancia de aprendizajes, en ocasiones alejados de los contenidos, relacionados con los valores que fomentan la creación de sentido, el respeto a los otros, la transformación. Las interacciones que se desarrollan en el aula fomentan el aprendizaje, la participación y la reflexión tal y como señala Bruner (1997).

\section{Relación con el entorno}

En el análisis de las intervenciones del alumnado durante las sesiones, un $18.0 \%$ de estas se basaban en las interacciones que realizan con su entorno, teniendo como referencia la lectura del libro. Por ejemplo, una alumna relaciona el concepto de invasión cultural (Freire 1970) con la violencia de género:

“Cuando esta tiene lugar, llega un momento que hay como dos roles, uno domina y otro dominado y que se aprovecha esa condición de sentirse inferior, en este caso la mujer, para que el otro se crea todavía más superior”. 
Otra alumna señala que ha comentado el libro leído con su familia y les ha dicho:

“Tenéis que leer este libro (...) siempre tienen mucha exigencia sobre ti. Cuando les dije que iba a estudiar esta carrera, me decían: ¿Pero no quieres ser profesora? Que no, que yo seré lo que quiera ser (...). Tienen unas expectativas para ti que tienes que lograr, porque eres una buena hija, pero son suyas".

Otra añade:

“Te preguntan: ¿Qué nota vas a sacar? No te preguntan si has aprendido. De forma inconsciente fomentan la competitividad".

Esta alumna se cuestiona que lo más importante para su familia sea la nota y no si se produce aprendizaje.

Estas respuestas muestran el interés de esta categoría para los estudiantes, no solo a nivel teórico sino también a nivel experiencial hablando de ello en las sesiones de las tertulias.

En las entrevistas se preguntaba sobre las interacciones con los compañeros de clase, con sus amistades y su familia. Todas las respuestas establecieron en mayor o menor medida que estas se producían. Algunas de las respuestas fueron:

“A mí sí me sorprenden las intervenciones de los demás, puedo llegar a conocerlos más profundamente (...) Me ha dado pie a hablar con otras personas que nunca pensé pasar de una relación basada en la educación: hola, buenos dias". "Al final son tus compañeras y compartes con ellas clase, pero hay temas que nunca te para a hablar con ellas. Salían temas que no habías hablado y pensabas: ¡Jo! mira esta, ¡qué guay! iqué idea ha dicho!

En cuanto a las interacciones con la familia y amigos, algunas respondían:

"Te dan ganas de transmitirlo a tus seres queridos lo que has aprendido y lo que te ha removido, es como que te gusta tener temas de conversación diferentes", "yo hago porque en mi casa se lean los libros que se leen aquí. En las comidas familiares no puedo hablar de otra cosa", "Yo a mi entorno le cuento de lo que hablamos en los seminarios, de la desigualdad", "Salía de los seminarios, iba a casa y a lo mejor estábamos comiendo: hoy hemos estado hablando de esto y ¿vosotros qué opináis? Creaba como mi debate en casa o con mis amigas", "Cuando leía y no entendía algo: 
mamá no entiendo esta frase. Se la leía y me decía: lo siento, yo tampoco, jes que Freire!".

Los libros, los debates en torno a ellos en las sesiones de las TPD, traspasaban las paredes del aula y continuaban en las casas de los estudiantes y entre sus amistades.

Estas respuestas muestran el interés de esta categoría para los estudiantes, no solo a nivel teórico sino también a nivel experiencial hablando de ello en las sesiones de las tertulias.

\section{Discusión y Conclusiones}

Los datos obtenidos de los estudiantes con los distintos instrumentos utilizados han estado en sintonía entre ellos a excepción de lo que respecta a la categoría actividad profesional y aprendizaje. En cuanto a la actividad profesional en el cuestionario, prácticamente la totalidad de los estudiantes $(96.6 \%)$ percibieron las TPD como un instrumento necesario para mejorar su práctica profesional, no fue así durante las sesiones de las tertulias, en las que solo hicieron referencia directamente a su futura actividad profesional en un $6.3 \%$ del total de las intervenciones realizadas. Al analizar las entrevistas en profundidad, evidenciamos que la totalidad de la submuestra consideró las TPD como una herramienta necesaria para implementar en su futura vida profesional, valorando la diversidad de colectivos con los que se podrían desarrollar. En este sentido, estos estudiantes coinciden con lo señalado por Flecha et. al (2014), señalando que las TPD les mejorará como futuros educadores sociales, señalando que favorecen: la igualdad, la escucha activa, la cohesión de grupo, el cambio social. Son conscientes de la heterogeneidad de la población con la que van a intervenir, implementando diferentes programas con el objetivo de mejorar su la calidad de vida. Estos estudiantes argumentan que la humildad es importante para su futuro trabajo en contextos en el que el diálogo es básico, pero un diálogo desde la igualdad, tal como señala Freire (1970).

La capacidad crítica y la reflexión han estado presentes a lo largo de toda la investigación y así se ha recogido en los distintos instrumentos utilizados. No solo percibían la reflexión, un $79.3 \%$, como un medio para el aprendizaje, sino que también fomentaba su transformación y la de su entorno. Ha favorecido las interacciones en el aula y la valoración de las opiniones de todos los componentes de las tertulias. Resaltan, por encima de otros valores, que toda opinión sea válida y que se expresen libremente como contrapunto a lo que se suele 
vivir en las sesiones de clase universitarias y de experiencias escolares anteriores En el ámbito universitario como señalan Rekalde et al., 2014, es importante crear espacios para la reflexión, para la crítica, para ser conscientes de la realidad en la que viven y ayudará a ser más competentes profesionalmente. En las entrevistas una alumna señala: creo que he mejorado como persona. Por otro lado, ha estado presente en las sesiones de las TPD, suponiendo un $63.5 \%$ del total de las intervenciones. Podemos señalar que la participación de todo el alumnado desde la igualdad favorece la reflexión y el espíritu crítico, como recogen Alarcón, Mora y Pérez, (2016). Por otro lado, un alumnado poco habituado, en general, a argumentar no intenta imponer sus ideas, las quiere compartir con los demás. "El debatirlo, yo creo que nos enriquece a todos".

El debate, la reflexión contribuye a la construcción del aprendizaje, tal como recogen García, Morlà y Ionescu (2018).

En aprendizaje, otra de las categorías establecidas, el alumnado percibe que esta práctica ha favorecido acceder a otra forma de aprender, basado en la escucha (89.6\%); en el aprendizaje entre todos (87.4\%). Una alumna señala: a veces leía un párrafo y no lo entendía, luego hablando con mis compañeras. ¡Ah!, pues sí, ahora lo entiendo.

En este sentido, Gutiérrez y Castro (2018) consideran que el aprendizaje entre iguales favorece su eficacia y aumenta las ganas de aprender. Aunque estos autores se refieren a un contexto educativo de una escuela, es extrapolable al contexto universitario, ya que también en este el aprendizaje se favorece cuando es entre iguales. Un aprendizaje alejado de los valores tradicionales y jerárquicos de la educación (Freire 1970). Descubren un aprendizaje basado en la igualdad de todos los participantes en las tertulias, sin imponer ninguna opinión (93.1\%) y el diálogo igualitario (95.42\%). Pero las intervenciones en las sesiones de TPD fueron un $8.4 \%$. Esta gran diferencia puede deberse a que durante el desarrollo de las sesiones se centraban en el relato de lo leído, comentando por qué lo habían elegido y escuchando las opiniones del resto de los componentes del grupo sin pararse a pensar en el aprendizaje que estaban experimentando. En cambio, en las entrevistas realizadas, como ya señalamos, la totalidad valora este aprendizaje, señalando aspectos como: Creo que es una forma de trabajar los contenidos que damos en clase que es diferente a lo común (...), y como que te enriqueces tanto del libro como de tus compañeros que forman las tertulias. Si el aprendizaje es importante en todos los niveles educativos en la universidad es básico; se están formando futuros profesionales, en un espacio europeo que implica cambios metodológicos, fomentando desde 
la práctica otros modelos igualitarios, reflexivos, participativos de aprendizaje, (Barba, 2013; Rekalde et al., 2014).

Tras estos años de experiencia con el TPD, podemos afirmar que la formación de los educadores sociales, a través de esta buena práctica, les proporciona competencias importantes como la escucha activa, respeto a los demás y aprendizaje entre todos que son herramientas fundamentales en su futuro profesional. Además, les supone un ejercicio de crítica y reflexión compartida que pocas veces se desarrolla tanto en las aulas universitarias.

La relación con el entorno: el alumnado nos indica en un $56.3 \%$ que relaciona lo leído con la actualidad, frente a un 2.2\% que no lo hace. Así mismo el contenido de las tertulias sale del aula y lo comentan con sus familias y amigos, reforzando las interacciones facilitadoras del aprendizaje (Bruner 1997). Tanto en las sesiones de las tertulias, como en las entrevistas se manifiesta este hecho, reforzándose las interacciones y mejorándolas (Ramis 2018). Estas aportaciones, de varias alumnas, ponen de manifiesto lo señalado: "Venimos juntas en el coche y vamos hablando de los libros, es el tema de conversación; un estudiante recuerda los consejos de su abuelo: "Tienes que estudiar para que no te laven el cerebro".

Otra alumna aporta: "En las comidas familiares no puedo hablar de otra cosa y recojo los argumentos de los compañeros y se los digo a mis padres”. Relacionar lo leído con su realidad social, familiar, personal facilita su comprensión. Como señalan (Freire, 1970; Gallardo-Pérez, 2014) el aprendizaje, en un contexto dialógico está relacionado con el entorno del alumnado y forma parte de su proceso educativo, fomentando la reflexión.

Queda mucho por hacer en educación superior, pero el desarrollo de las capacidades relacionadas con la relación con los demás, trabajo en equipo, respeto, igualdad, solidaridad son valores que hay que trabajar desde las aulas en los distintos grados universitarios y con muestras mayores.

La falta de estudios de esta buena práctica en la enseñanza universitaria nos muestra la necesidad de llevar a cabo más experiencias relacionadas con buenas prácticas de éxito que nos aseguren una educación superior que desarrolle las competencias adecuadas y donde los estudiantes, además de aprender su profesión, compartan, escuchen, aporten y transformen.

Por último, señalar que este artículo no hubiera sido posible sin las voces de nuestro alumnado, a todos ellos gracias por sus reflexiones, por ratificarnos que otra forma de aprender es posible y que todos tenemos más fuerza para avanzar. 


\section{Referencias}

Alarcón, O., Mora, H. y Pérez, J.A. (2016). La tertulia pedagógica, una estrategia dialógica potenciadora de la comprensión lectora y de las interacciones docentes. Revista Oralidad-es, 2 (4), 32-41.4 https://revistaoralidad-es.com/index.php/ro-es/article/view/51

Aguilar Ródenas, C. (2017). La Tertulia pedagógica en el prácticum de la formación inicial de maestros y maestras. Revista Iberoamericana de Educación vol. 73, núm.2. p.922. https://doi.org/10.35362/rie732198

Aguilera Jiménez, A., Prados Gallardo, Mª M. y Gómez del Castillo Segurado, Ma T. (2015). La experiencia del voluntariado universitario en las tertulias dialógicas de las comunidades de aprendizaje. Revista de Docencia Universitaria REDU, 13 (2), 249267. https://doi.org/10.4995/redu.2015.5447

Álvarez Álvarez, C., González Cotado, L. y Larrinaga Iturriaga, A. (2012). Aprendizaje dialógico, grupos interactivos y tertulias literarias: Una apuesta de centro educativo que favorece la inclusión. V Congreso Mundial de Estilos de Aprendizaje, Santander.

Aubert, A., Flecha, A., García, C., Flecha, R., \& Racionero, S. (2008). Aprendizaje dialógico en la Sociedad de la Información. Barcelona: Hipatia.

Bonell García, L.; Martínez-González, A.; Rodríguez-Fernández, A. (2019). Tertulias dialógicas en los estudios de Educación Social y trabajo Social del centro Universitario la Salle. Revista Indivisa, Boletín de estudios e Investigaciones, 19. 137-161

Bruner, J. (1997). La educación puerta de la cultura. Madrid: Antonio Machado Libros.

Chocarro de Luis, E. (2013) Tertulias dialógicas, un recurso didáctico en la formación de docentes. Historia y Comunicación Social. Vol. 18. № Esp. nov. 219-229. http://dx.doi.org/10.5209/rev_HICS.2013.v18.44238

Del Pino-Sepúlveda, M; Del Pino-Sepúlveda, A y Pinchira-Fuentealba, D. (2016). La lectura desde el enfoque del Ministerio de Educación y el enfoque dialógico de la pedagogía “Enlazando mundos". Revista Electrónica Educar, 2 (3) 1-2. http://dx.doi.org/10.15359/ree.20-3.6

Flecha, R. (1997). Compartiendo palabras. Barcelona: Paidós 
Flecha, R. (Ed.) (2015). INCLUD-ED. Consortium, successful educational actions for inclusion and social cohesion en Europe. Springer Briefs in Education.

doi 10.1007/978-3-319-11176-6

Flecha, R., Racionero, S., Tintoré, M., y Arbós, A. (2014). Actuaciones de Éxito en la Universidad. Hacia la Excelencia Tomando las Mejores Universidades como Modelo. Multidisciplinary Journal of Educational Research, 4 (2), pp. 131-150. doi: $10.4471 /$ remie. 2014.08

Freire, P. (1970). Pedagogía del Oprimido. Madrid: siglo XXI. $1^{\text {a }}$ ed. 1970.

Freire, P. (1997). A la sombra de este árbol. Barcelona: El Rore Editorial

Gallardo-Pérez, H. de J. (2014). El modelo pedagógico dialógico crítico en la educación. Respuestas, 19(2), 81-92. https://doi.org/10.22463/0122820X.498

García-Carrión, R; López de Aguileta, G; Padrós, M \& Ramis-Salas, M. (2020). Implicaciones para el impacto social de la enseñanza y el aprendizaje dialógico. Phychol. https://doi.org/10.3389/fpsyg.2020.00140

Garcia Yeste, C.; Morlà Folch, T. y Ionescu, V. (2018). Sueños de educación superior en la escuela Mediterrani a través de la educación familiar. Frente. Educ. https://doi.org/10.3389/feduc.2018.00079

Grañeras, M., Gil, N y Díaz-Caneja, P. (2011). Actuaciones de éxito en las escuelas europeas (vol. 9). Ministerio de Educación.

Gómez, J., Latorre, A., Sánchez, M. \& Flecha, R. (2006). Metodología comunicativa crítica. Barcelona: El Roure Ciencia.

Gutiérrez Esteban, P; Castro Robles, Mª. (2018). El aprendizaje entre iguales como metodología de trabajo para la inclusión educativa. Experiencia docente en una escuela de Extremadura. Revista de Investigación en Educación, [S.1.], v. 16, n. 1, p. 78-92. http://reined.webs.uvigo.es/index.php/reined/article/view/318

Habermas, J. (1992). Teoría de la acción comunicativa, II. Madrid: Taurus Humanidades.

Malagón Moreno, JD y González López, I. (2018). Evaluación del impacto de las tertulias literarias dialógicas en comunidades de aprendizaje. Revista de currículum y formación del profesorado, 22 (4) 111-132.

doi:10.30827/profesorado.v22i4.8400 
Ortega Palacios, I y Álvarez Álvarez, C. (2015). Cuatro años de grupos interactivos: estudio de caso de un centro educativo pionero. Educatio Siglo XXI, Vol. 33 n 2 - 2015, pp. 105-122 105

http://dx.doi.org/10.6018/j/232711

Palomares-Montero, D. y Gabaldón-Estevan, D. (2016). Tertulias dialógicas y grupos interactivos en el ámbito de la docencia universitaria. Una revisión del estado del arte. Llibre dàctes de IV Jornades Ides 2015. Universitat de Valéncia, pp. 155-168-

Ramis, M. (2018). Contribuciones de la Teoría de Freire a la Educación Dialógica. HSE Social and Education History. Vol. 7 No. 3 October 2018 pp. 277-299 doi: $10.1783 /$ hse. 2018.3749

Rekalde Rodríguez, I., Alonso Olea, j., Arandia Loroño, M., Martínez Domínguez, I. y Zarandona de Juanes, E. (2014). Las tertulias literarias dialógicas en los procesos de enseñanza universitarios: Reflexiones desde la práctica docente. Revista de Formación e Innovación Educativa Universitaria. Vol. 7, № 3, 155-172

Roca Campos, E. (2016). Formación dialógica del profesorado. Reencanto con la profesión docente. Padres y Maestros, 387, 11-16 doi: pym.i367.y2016.002 